Pacific Journal of Mathematics

CAUCHY SPACES WITH REGULAR COMPLETIONS

ell Conley Kent and Gary Douglas Richardson 


\title{
CAUCHY SPACES WITH REGULAR COMPLETIONS
}

\author{
D. C. Kent and G. D. Richardson
}

\begin{abstract}
A $T_{3}$ Cauchy space which has a regular completion is shown to have a $T_{3}$ completion, but an example shows that such Cauchy spaces need not have strict $T_{3}$ completions. Various conditions are found for the existence of $T_{3}$ completions and strict $T_{3}$ completions; for instance, every Cauchy-separated, locally compact, $T_{3}$ Cauchy space has a $T_{3}$ completion. Convergence spaces and topological spaces which have a coarsest compatible Cauchy structure with a strict $T_{3}$ completion are characterized, as are those spaces for which every compatible $T_{3}$ Cauchy structure has a $T_{3}$ completion.
\end{abstract}

Introduction. Cauchy spaces were first defined in their present form by $H$. Keller [3] in 1968. Most subsequent work in this area has dealt with Cauchy space completions; the study of regular completions was initiated by J. Ramaley and O. Wyler [9] in 1970. Cauchy spaces are finding applications in various areas; they were applied in the study of $C^{*}$-algebras by K. McKennon [8], and recently some very nice results on Cauchy completions of lattice ordered groups were obtained by R. Ball [1].

At the present time, the two most important unsolved problems in the study of Cauchy spaces are:

(1) Find a completion functor for Cauchy groups (or prove that none exists).

(2) Find internal (and usable) characterizations of Cauchy spaces which have regular completions.

This paper attacks the second problem by expanding on ideas introduced in [5] and [7].

The first section is concerned with " $C_{3}$ Cauchy spaces," which is the name we give to Cauchy spaces that have $T_{3}$ completions. An early result is that a $T_{3}$ Cauchy space which has a regular (non- $T_{2}$ ) completion also has a $T_{3}$ completion. This result is surprising in view of the very different behavior of regular and $T_{3}$ compactifications studied in [6]. We also obtain useful criteria for locally compact Cauchy spaces to be $C_{3}$.

In the second section, we give an example of a $C_{3}$ Cauchy space which has no strict $T_{3}$ completion. Thus Problem (2) splits into two problems, the second being to characterize those Cauchy spaces which have strict $T_{3}$ completions (we call these $S C_{3}$ Cauchy spaces). The solution which we give to the latter problem (see Proposition 2.3) is useful, but not entirely satisfying. 
In $\S 3$ we shift our attention to $T_{3}$ convergence spaces and pose two questions:

(1) When does a $T_{3}$ convergence space have a coarsest compatible $C_{3}$ (or $\mathrm{SC}_{3}$ ) Cauchy structure?

(2) When are all compatible $T_{3}$ Cauchy structures $C_{3}$ (or $S C_{3}$ ) Cauchy structures?

In answering these questions we introduce two new convergence space notions which we call " $r$-boundedness" and " $s$-boundedness." The former is closely related to local compactness, the latter bears some resemblance to countable compactness. The $r$-bounded convergence spaces provide the answer to Question (1); spaces which have both properties (we call these $r s$-bounded spaces) are the answer to Question (2).

1. $C_{3}$ Cauchy spaces. For basic definitions and terminology pertaining to Cauchy spaces and convergence spaces, the reader is asked to refer to [5]. A few changes in the notation of [5] will be made; most notably, we will use " $\mathrm{cl}_{q}$ " instead of " $\Gamma_{q}$ " for the closure operator of a convergence space $(X, q)$, and we shall denote the set of Cauchy equivalence classes of a Cauchy space $(X, \mathcal{C})$ by $X^{*}$. The set of all filters on a set $X$ will be denoted by $F(X)$.

A convergence space $(X, q)$ is said to be $T_{2}$ if each filter converges to at most one point; $(X, q)$ is regular if $\mathrm{cl}_{q} \mathscr{F} \rightarrow x$ whenever $\mathscr{F} \rightarrow x$. A regular $T_{2}$ convergence space is said to be $T_{3}$. A regular convergence space is defined to be symmetric if $\mathscr{F} \rightarrow x$ whenever $\mathscr{F} \rightarrow y$ and $\dot{y} \rightarrow x ; T_{3}$ spaces are obviously symmetric. A convergence space is locally compact if every convergent filter contains a compact set.

A Cauchy space $(X, \mathcal{C})$ is defined to be $T_{2}$ or locally compact if the induced convergence structure $q_{e}$ has the same property. On the other hand, a Cauchy space $(X, \mathcal{C})$ is regular if $\mathrm{cl}_{q_{\mathcal{C}}} \mathscr{F} \in \mathcal{C}$ whenever $\mathscr{F} \in \mathcal{C}$; if $(X, \mathcal{C})$ is regular then so is $\left(X, q_{e}\right)$, but the converse is false. Like a convergence space, a Cauchy space is said to be $T_{3}$ if it is both regular and $T_{2}$. This paper is devoted to the study of Cauchy spaces which have $T_{3}$ completions; we shall call these $C_{3}$ Cauchy spaces.

Proposition 1.1. If $(X, \mathcal{C})$ is a regular Cauchy space, then the induced convergence space $\left(X, q_{\mathcal{e}}\right)$ is symmetric.

Let $(X, \mathcal{C})$ be a $T_{2}$ Cauchy space. If $\mathscr{F} \in \mathcal{C}$ let $[\mathscr{F}]=\{\mathcal{G} \in \mathcal{C}: \mathscr{F} \cap \mathcal{G}$ $\in \mathcal{C}\}$ denote the Cauchy equivalence class containing $\mathscr{F}$. Let $X^{*}=\{[\mathscr{F}]:$ $\mathscr{F} \in \mathcal{C}\}$, and let $j: X \rightarrow X^{*}$ be the natural injection $j(x)=[\dot{x}]$, all $x \in X$. 
A completion of $(X, \mathcal{C})$ which has $X^{*}$ as its underlying set and $j$ as its embedding map such that $j \mathscr{F} \rightarrow[\mathscr{F}]$ for each $\mathscr{F} \in \mathcal{C}$ is said to be in standard form. Reed [10] has shown that every $T_{2}$ completion of $(X, \bigodot)$ is equivalent to one in standard form.

A complete Cauchy structure $\complement^{*}$ on $X^{*}$ is given by: $\bigodot^{*}=\left\{\mathscr{Q} \in F\left(X^{*}\right)\right.$ : $\mathbb{Q} \geq j(\mathscr{F}) \cap[\dot{\mathscr{F}}]$, for some $\mathscr{F} \in \mathcal{C}\}$. If $(X, \mathcal{C})$ is $T_{2}$, then $\left(X^{*}, \mathcal{C}^{*}\right)$ is also $T_{2}$, and $\left(\left(X^{*}, \bigodot^{*}\right), j\right)$ is called Wyler's completion of $(X, \mathcal{C})$. It is easy to see that if $\left(\left(X^{*}, \mathscr{D}\right), j\right)$ is any other $T_{2}$ completion of $(X, \mathcal{C})$ in standard form, then $e^{*} \subseteq \mathcal{D}$; in other words, Wyler's completion is (up to equivalence) the finest completion of any $T_{2}$ Cauchy space.

For any Cauchy space $(X, \mathcal{C})$ let $\mathcal{C}_{r}$ be the finest regular Cauchy structure coarser than $C$. It is easy to verify that $e_{r}$ is complete whenever $e$ is complete. If $q=q_{e}$ is the convergence structure induced by $e$, then it follows easily from Proposition 1.1 that the convergence structure on $X$ induced by $e_{r}$ is $\sigma q$, the finest symmetric convergence structure coarser than $q$. In what follows we shall denote by $q^{*}$ (respectively, $\sigma q^{*}$ ) the convergence structure on $X^{*}$ induced by $\mathcal{C}^{*}$ (respectively, $\mathcal{C}_{r}^{*}$ ).

Proposition 1.2. If a $T_{2}$ Cauchy space $(X, \mathcal{C})$ has a regular completion, then $\left(\left(X^{*}, \bigodot_{r}^{*}\right), j\right)$ is a regular completion of $(X, \mathcal{C})$. Furthermore, in this case, $\left(\left(X^{*}, \mathcal{C}_{r}^{*}\right), j\right)$ is a $T_{3}$ completion of $(X, \mathcal{C})$.

Proof. Let $\left(\left(X^{*}, \mathscr{Q}\right), j\right)$ be a regular completion of $(X, \mathcal{C})$ in standard form. Then $\mathscr{Q} \leq \mathfrak{C}_{r}^{*} \leq \mathfrak{C}^{*}$, and it follows immediately that $j:(X, \mathcal{C}) \rightarrow$ $\left(X^{*}, \bigodot_{r}^{*}\right)$ is a Cauchy embedding, which proves the first assertion. To prove the second, note that $[\dot{\mathscr{F}}] \cap[\dot{\mathcal{G}}] \in \mathcal{C}_{r}^{*}$ implies $\left(\mathrm{cl}_{\sigma q^{*}} j \mathscr{F}\right) \vee$ $\left(\mathrm{cl}_{\sigma q^{*}} j \mathcal{G}\right) \neq \varnothing$, so

$$
\left(\mathrm{cl}_{\sigma q^{*}} j \mathscr{F}\right) \cap\left(\mathrm{cl}_{\sigma q^{*}} j \mathcal{G}\right)=\mathrm{cl}_{\sigma q^{*}} j(\mathscr{F} \cap \mathcal{G}) \in \mathcal{C}_{r}^{*} .
$$

Hence, $\mathscr{F} \cap \mathcal{G} \in \mathcal{C}$, and $[\mathcal{F}]=[\mathcal{G}]$. It follows that $\left(X^{*}, \mathcal{C}_{r}^{*}\right)$ is $T_{2}$ and, hence, $T_{3}$.

COROLlARY 1.3. The following statements about a $T_{3}$ Cauchy space are equivalent.

(1) $(X, \mathcal{C})$ is $C_{3}$.

(2) $(X, \mathcal{C})$ has a regular completion.

(3) $\left(\left(X^{*}, \bigodot_{r}^{*}\right), j\right)$ is a $T_{3}$ completion of $(X, \mathcal{C})$.

When one compares regular versus $T_{3}$ compactifications of $T_{3}$ convergence spaces, the results differ significantly from those obtained for 
Cauchy completions in Corollary 1.3. In a recent paper [6], we showed that the $T_{3}$ spaces having regular compactifications are precisely the $\omega$-regular convergence spaces, whereas those having $T_{3}$ compactifications constitute the proper subclass of completely regular spaces. The apparent discrepancy is due to the fact that regular compactifications, unlike regular Cauchy completions, need not be symmetric. Convergence spaces which are $\omega$-regular, but not completely regular, have non-symmetric regular compactifications which cannot be constructed via Cauchy completions.

Lemma 1.4. If $(X, \mathcal{C})$ is locally compact and $T_{3},\left(X^{*}, \mathrm{C}_{r}^{*}\right)$ is $T_{2}$, and $A \subseteq X^{*}$, then $j^{-1}\left(\mathrm{cl}_{\sigma q^{*}} A\right)=\mathrm{cl}_{q} j^{-1}(A)$.

Proof. Let $q=q_{\mathrm{e}}$ be the convergence structure induced by $\mathrm{C}$. The continuity of $j$ implies $\mathrm{cl}_{q} j^{-1}(A) \subseteq j^{-1}\left(\operatorname{cl}_{\sigma q^{*}} A\right)$. If $x \in j^{-1}\left(\operatorname{cl}_{\sigma q^{*}} A\right)$, then there is a filter $\mathbb{Q} \rightarrow[\dot{x}]$ in $\left(X^{*}, \sigma q^{*}\right)$ such that $A \in \mathbb{Q}$. It follows that $\mathbb{Q} \geq \operatorname{cl}_{\sigma q^{*}}^{n}(j(\mathscr{F}) \cap[\dot{\mathscr{F}}])$ for some $n \in N$ and $\mathscr{F} \in \mathcal{C}$. Since $\left(X^{*}, \mathcal{C}_{r}^{*}\right)$ is $T_{2}$, $[\mathcal{F}]=[\dot{x}]$, so $\mathbb{Q} \geq \operatorname{cl}_{\sigma q^{*}}^{n} j(\mathscr{F} \cap \dot{x})$, where $\mathscr{F} \cap \dot{x} \in \mathcal{C}$. Since $(X, \mathcal{C})$ is locally compact and $T_{3}, \operatorname{cl}_{q}(\mathscr{F} \cap \dot{x})$ has a base of compact sets. Using the fact that $\left(X^{*}, \mathcal{C}_{r}^{*}\right)$ is also $T_{3}$, it follows that $\mathrm{cl}_{\sigma q^{*}}^{n} j(\mathscr{F} \cap \dot{x})=j\left(\operatorname{cl}_{q}(\mathscr{F} \cap \dot{x})\right)$, so $\mathbb{Q} \geq j\left(\mathrm{cl}_{q}(\mathscr{F} \cap \dot{x})\right)$. The filter $\mathrm{cl}_{q}(\mathscr{F} \cap \dot{x})$ thus has a trace $\mathcal{G}$ on $j^{-1}(A)$ which $q$-converges to $x$, and we conclude that $x \in \mathrm{cl}_{q} j^{-1}(A)$.

THEOREM 1.5. A locally compact, $T_{3}$ Cauchy space $(X, \mathcal{C})$ is $C_{3}$ iff $\left(X^{*}, \mathrm{C}_{r}^{*}\right)$ is $T_{2}$.

Proof. It is obvious that $j:(X, \mathcal{e}) \rightarrow\left(X^{*}, \mathrm{C}^{*}\right)$ is Cauchy-continuous and one-to-one. If $\left(X^{*}, \bigodot_{r}^{*}\right)$ is $T_{2}$, then, by the preceding lemma, $j^{-1}\left(\operatorname{cl}_{\sigma q^{*}}^{n}(j \mathcal{G})\right)=\operatorname{cl}_{q}^{n} \mathcal{G}$ for all $n \in N$ and $\mathcal{G} \in \mathcal{C}$; this is precisely what is needed to show that $j^{-1}:\left(j X,\left.\mathcal{C}_{r}^{*}\right|_{j X}\right) \rightarrow(X, \mathcal{C})$ is Cauchy-continuous, and it follows that $\left(X^{*}, \mathcal{C}_{r}^{*}\right)$ is a $T_{3}$ completion of $(X, \mathcal{C})$. The converse follows immediately from Proposition 1.2.

Given a $T_{2}$ Cauchy space $(X, \mathcal{C})$, let $\hat{C}(X, \mathcal{C})$ be the set of all Cauchy-continuous functions from $(X, \mathcal{C})$ into the set $R$ of real numbers with the usual (complete) Cauchy structure. A $T_{2}$ Cauchy space $(X, \mathcal{C})$ is said to be Cauchy separated if the set $(X, \mathcal{C})$ separates Cauchy equivalence classes; i.e., if $\mathscr{F}, \mathcal{G} \in \mathcal{C}$ and $\mathscr{F} \cap \mathcal{G} \notin \mathcal{C}$ implies there is $f \in \mathcal{C}(X, \mathcal{C})$ such that $f(\mathscr{F})$ and $f(\mathcal{G})$ converge to distinct points in $R$.

Lemma 1.6. If $(X, \mathcal{C})$ is Cauchy-separated, then $\left(X^{*}, \mathcal{C}_{r}^{*}\right)$ is $T_{2}$. 


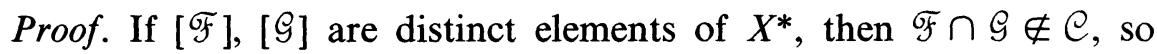
there is $f \in \hat{C}(X, \mathcal{C})$ such that $\lim f(\mathscr{F}) \neq \lim f(\mathcal{G})$ in $R$. By Proposition 2.1, [2], $f$ has a continuous extension $\bar{f}:\left(X^{*}, \bigodot^{*}\right) \rightarrow R$, and, since $R$ is regular, $\bar{f}:\left(X^{*}, \mathcal{C}_{r}^{*}\right) \rightarrow R$ is also continuous. But $\bar{f}([\mathcal{F}])=\lim f(\mathscr{F}) \neq$ $\lim f(\mathcal{G})=\bar{f}([\mathcal{G}])$, and the conclusion is established.

An immediate consequence of Lemma 1.6 and Theorem 1.5 is the following.

TheOREM 1.7. A regular, Cauchy separated, locally compact Cauchy space is $C_{3}$.

THEOREM 1.8. A totally bounded $T_{3}$ Cauchy space $(X, \mathcal{C})$ is $C_{3}$ iff $(X, \mathcal{C})$ is Cauchy separated.

Proof. If $(X, \mathcal{C})$ is $C_{3}$, then $\left(X^{*}, \bigodot_{r}^{*}\right)$ is a $T_{3}$ compactification of $X$, which means that $\left(X^{*}, \mathcal{C}_{r}^{*}\right)$ has the same ultrafilter convergence as a Tychonoff topological space. Since each continuous real-valued function on $\left(X^{*}, \bigodot_{r}^{*}\right)$, when restricted to the image of $(X, \mathcal{C})$, is Cauchy-continuous, it follows that $(X, \mathcal{C})$ is Cauchy-separated. The converse follows from Theorem 2.2 [5].

Can the assumptions made in the above theorems be weakened? We have an example (omitted for the sake of brevity) of a $T_{3}$ Cauchy space $(X, \mathcal{C})$ such that $\left(X^{*}, \mathrm{C}_{r}^{*}\right)$ is $T_{2}$ but is not a completion of $(X, \mathcal{C})$; thus the assumption of local compactness in Theorem 1.5 cannot be entirely dispensed with. We also have an example of a locally compact, $T_{3}$ Cauchy space which is not $C_{3}$, so "Cauchy separated" cannot be replaced by " $T_{2}$ " in Theorem 1.7. On the other hand, a locally compact $C_{3}$ Cauchy space need not be Cauchy-separated. For it should be noted that any $T_{3}$ convergence space is a (complete) $C_{3}$ Cauchy space. In Example 2.10, [4], a locally compact, $T_{3}$ convergence space is constructed whose topological modification is not $T_{2}$; this space, when regarded as a complete Cauchy space, is clearly not Cauchy separated.

2. $S C_{3}$ Cauchy spaces. A completion $((Y, \mathscr{D}), h)$ of a Cauchy space $(X, \mathcal{C})$ is defined to be strict if, whenever $\mathcal{Q} \in \mathcal{W}$, there is $\mathscr{F} \in \mathcal{C}$ such that $\mathbb{Q} \geq \mathrm{cl}_{q_{\odot}} h(\mathscr{F})$. This terminology was introduced in [5], where it was shown that every $T_{2}$ Cauchy space has a coarsest strict (not necessarily regular or $T_{2}$ ) completion in standard form. 
Topological completions are necessarily strict; also, if $(X, \mathcal{C})$ is a $T_{2}$ Cauchy space such that $X^{*}-j(X)$ is finite, then any completion of $(X, \mathcal{C})$ is strict. On the other hand, $T_{3}$ completions need not be strict. When they exist, however, strict $T_{3}$ completions are unique up to equivalence (see [5]). One of the main results of this section is an example of a locally compact Cauchy space which has a $T_{3}$ completion but no strict $T_{3}$ completion. Thus the Cauchy spaces having strict $T_{3}$ completions form a proper subclass of the $C_{3}$ Cauchy spaces; a member of the former class will be called an $\mathrm{SC}_{3}$ Cauchy space.

To facilitate our study of $S C_{3}$ Cauchy spaces, we shall make use of the " $\Sigma$ operator" defined in [5] for a $T_{2}$ Cauchy space $(X, \mathcal{C})$ as follows. If $A \subseteq X$, let $\Sigma A=\left\{[\mathscr{F}] \in X^{*}: A \in \mathcal{G}\right.$ for some $\left.\mathcal{G} \in[\mathscr{F}]\right\}$. If $\mathscr{F} \in F(X)$, let $\Sigma \mathscr{F}$ be the filter on $X^{*}$ generated by $\{\Sigma F: F \in \mathscr{F}\}$. Let $\mathcal{C}_{1}^{*}$ be the Cauchy structure on $X^{*}$ generated by $\{\Sigma \mathscr{F}: \mathscr{F} \in \mathcal{C}\}$, and let $q_{1}^{*}$ be the induced convergence structure. (In [5] $q_{1}^{*}$ was denoted by $p$ and $\bigodot_{1}^{*}$ by $\bigodot_{p}$.) From the construction, it is clear that $\mathrm{C}_{r}^{*} \leq \mathfrak{C}_{1}^{*} \leq \mathrm{e}^{*}$.

Proposition 2.1. Let $(X, \mathcal{C})$ be a $T_{3}$ Cauchy space. Then $(X, \mathcal{C})$ is $S C_{3}$ iff $\bigodot_{1}^{*}=\bigodot_{r}^{*}$.

Proof. Corollary 1.6, [5], asserts that $\left(X^{*}, \bigodot_{1}^{*}\right)$ is the only possible strict $T_{3}$ completion of $(X, \mathcal{C})$ in standard form. If $(X, \mathcal{C})$ is $S C_{3}$, then $e_{1}^{*}=e_{r}^{*}$. Conversely, if $e_{1}^{*}=e_{r}^{*}$, then $\left(\left(X^{*}, \varrho_{r}^{*}\right), j\right)$ is a strict completion of $(X, \mathcal{C})$ by the results of [5]; it then follows from Proposition 1.2 that $\left(\left(X^{*}, e_{r}^{*}\right), j\right)$ is a strict $T_{3}$ completion of $(X, \mathcal{C})$.

Theorem 1.5 shows that every locally compact $T_{3}$ Cauchy space for which $\left(X^{*}, \mathcal{C}_{r}^{*}\right)$ is $T_{2}$ is a $C_{3}$ Cauchy space. The next example shows that such spaces need not be $S C_{3}$.

EXAMPle 2.2. Let $X$ be an infinite set partitioned into infinite subsets $\left\{X_{n}: n=0,1,2, \ldots\right\}$. Let $\mathscr{F}$ be a free filter on $X$ which contains $X_{0}$ and has a nested filter base $\left\{F_{n}: n \in N\right\}$ such that $F_{n}-F_{n+1}$ is an infinite set for $n \geq 1$. Furthermore, let each set $F_{n}-F_{n+1}$ be partitioned into infinite subsets $\left\{H_{n, k}: k \in N\right\}$, and let $\mathcal{H}_{n, k}$ be a free filter on $X$ which contains $H_{n, k}$ for all $n, k \in N$.

Next, for each $n \in N$, let $\mathcal{G}_{n}$ be a free filter on $X$ which contains $X_{n}$ and which has a nested base $\left\{G_{n, k}: k \geq 1\right\}$ such that $G_{n, k} \subseteq X_{n}$ and $G_{n, k}-G_{n, k-1}$ is an infinite set for all $n \geq 1$. Furthermore, for all $n$, $k \in N$, let $\varrho_{n, k}$ be a free filter on $X$ which contains $G_{n, k}-G_{n, k+1}$. 
Let $\mathcal{C}=\left\{\dot{x}, \mathscr{F}, \mathcal{G}_{n}, \mathscr{K}_{n, k}: x \in X, \mathscr{K}_{n, k} \geq \mathcal{H}_{n, k} \cap \mathcal{L}_{n, k}, n \in N, k \in N\right\}$. Then $q_{e}$ is discrete, and it follows easily that $(X, \mathcal{C})$ is locally compact and $T_{3}$. One can also show that $\left(X^{*}, \mathrm{C}_{r}^{*}\right)$ is $T_{2}$, so by Theorem $1.5(X, \mathcal{C})$ is a $C_{3}$ Cauchy space. To show that $(X, \mathcal{C})$ has no strict $T_{3}$ completion it is sufficient, by Proposition 2.1, to show that $\varrho_{1}^{*} \neq \mathcal{C}_{r}^{*}$. This can be accomplished by showing that $\mathrm{cl}_{q_{1}^{*}} j(\mathscr{F}) \neq \mathrm{cl}_{\sigma q^{*}} j(\mathscr{F})$. Indeed, each member of the latter filter contains elements of the form $\left[\mathcal{G}_{n}\right]$, and this is not true of the former.

In order to characterize $S C_{3}$ Cauchy spaces, we shall extend the $\Sigma$ operator. Given a $T_{2}$ Cauchy space $(X, \mathcal{C})$ and $A \subseteq X$, define $\Sigma^{2} A=\{[\mathscr{F}]$ $\in X^{*}$ : there is $\mathcal{G} \in[\mathcal{F}]$ such that $(\Sigma G) \cap(\Sigma A) \neq \varnothing$ for all $\left.G \in \mathcal{G}\right\}$. If $\mathscr{F} \in F(X)$, define $\Sigma^{2} \mathscr{F}$ to be the filter on $X^{*}$ generated by $\left\{\Sigma^{2} F: F \in \mathscr{F}\right\}$.

Proposition 2.3. Let $(X, \mathcal{C})$ be a $T_{2}$ Cauchy space. Then $(X, \mathcal{C})$ is $S C_{3}$ iff both of the following conditions are satisfied: (1) If $\mathscr{F}, \mathcal{G} \in \mathcal{C}$ and $(\Sigma \mathscr{F}) \vee(\Sigma \mathcal{G}) \neq \varnothing$, then $[\mathscr{F}]=[\mathcal{G}]$; (2) If $\mathscr{F} \in \mathcal{C}$, there is $\mathcal{G} \in \mathcal{C}$ such that $\Sigma^{2} \mathcal{F} \geq \Sigma \mathcal{G}$.

Proof. Assume the two conditions. The first guarantees that the space ( $X^{*}, \mathcal{C}_{1}^{*}$ ) is $T_{2}$. Since $\Sigma$ is the closure operator for the convergence structure $q_{1}^{*}$, the second condition guarantees that $\left(X^{*}, \bigodot_{1}^{*}\right)$ is regular. Thus $\mathrm{C}_{1}^{*}=\mathrm{C}_{r}^{*}$, and $(X, \mathcal{e})$ is $S C_{3}$ by Proposition 2.1 . Conversely, the two conditions follow directly from the assumption that $\mathcal{C}_{1}^{*}$ is $T_{3}$.

Although we have seen that locally compact $C_{3}$ Cauchy spaces are not necessarily $\mathrm{SC}_{3}$ spaces, one can obtain the following partial result.

Proposition 2.4. If $(X, C)$ is a locally compact $C_{3}$ Cauchy space, and $\mathbb{Q} \rightarrow[\dot{x}]$ in $\left(X^{*}, \mathfrak{C}_{r}^{*}\right)$, where $x \in X$, then there is $\mathscr{F} \in \mathcal{C}$ such that $\mathbb{Q} \geq$ $\mathrm{cl}_{\sigma q^{*}} j(\mathscr{F})$.

Proof. This result is implicit in the proof of Lemma 1.4.

The preceding proposition asserts that for locally compact $C_{3}$ spaces, "strictness" can fail only at "new" points which are added in the completion process.

We conclude this section by mentioning a class of locally compact $T_{3}$ Cauchy spaces which are a subclass of the $S C_{3}$ Cauchy spaces. A Cauchy space $(X, \mathcal{C})$ is called a sequential Cauchy space if every Cauchy filter 
contains a Cauchy filter which is generated by a sequence. A $T_{2}$ sequential Cauchy space is locally compact and $T_{3}$; furthermore, it is easy to verify that Wyler's completion preserves both of these properties. Thus if $(X, \mathcal{C})$ is a $T_{2}$ sequential Cauchy space, then $\left(X^{*}, \complement^{*}\right)=\left(X^{*}, \bigodot_{1}^{*}\right)=\left(X^{*}, \bigodot_{r}^{*}\right)$; the next proposition is an immediate consequence.

Proposition 2.5. A $T_{2}$ sequential Cauchy space is $S C_{3}$.

3. Coarse $C_{3}$ Cauchy spaces. Let $(X, q)$ be a $T_{3}$ convergence space, and let $[q]$ denote the set of all Cauchy structures on $X$ compatible with $q$. It is shown in [7] that [q] always contains a finest $T_{3}$ member, denoted by $e^{q}$, and a coarsest $T_{3}$ member, denoted by $\mathscr{Q}^{q}$. $\complement^{q}$ is complete, and is therefore the finest $C_{3}$ member of $[q]$. On the other hand, $Q^{q}$ is not $C_{3}$ in general; necessary and sufficient conditions for the existence of a coarsest $C_{3}$ member of $[q]$ are obtained below. We also characterize those $T_{3}$ spaces $(X, q)$ such that each $T_{3}$ member of $[q]$ is $C_{3}$.

Starting with a $T_{3}$ convergence space $(X, q)$ let $\complement^{q}$ be the set of all $q$-convergent filters on $X$. Let $\Delta_{q}=\left\{\mathscr{F} \in F(X): \mathcal{G} \vee\left(\operatorname{cl}_{q}^{n} \mathscr{F}\right)=\varnothing\right.$ for all $\mathcal{G} \in \mathcal{C}^{q}$ and $\left.n \in N\right\}$. Let $\mathscr{D}_{q}=\mathcal{C}^{q} \cup \Delta_{q}$, and let $\mathfrak{N}_{q}=\cap \Delta_{q}$. A $T_{3}$ convergence space $(X, q)$ is defined to be $r$-bounded if, for each $\mathscr{F} \in \mathcal{C}^{q}$, $\mathscr{F} \vee \Re_{q}=\varnothing$.

LEMMA 3.1. If $\left(X, \mathscr{D}_{q}\right)$ has a $T_{3}$ completion, then $(X, q)$ is $r$-bounded.

Proof. Let $(X, q)$ be a $T_{3}$ space which is not $r$-bounded. First note that $\Delta_{q}$ consists of a single Cauchy equivalence class of non-convergent filters; thus any completion of $\left(X, \mathscr{D}_{q}\right)$ is necessarily a one-point completion. If $\left(\left(X^{*}, p\right), j\right)$ is such a completion in standard form, let $\alpha=[\mathscr{F}]$, where $\mathscr{F} \in \Delta_{q}$; then $X^{*}=\{[\dot{x}]: x \in X\} \cup\{\alpha\}$, and if $\mathcal{G} \in \Delta_{q}$ then $j(\mathcal{G}) \rightarrow \alpha$ in $\left(X^{*}, p\right)$. Since $(X, q)$ is not $r$-bounded, there is $\mathscr{F} \rightarrow x$ in $(X, q)$ such that $\mathscr{F} \vee \mathscr{N}_{q} \neq \varnothing$; this implies that each $F \in \mathscr{F}$ belongs to some ultrafilter $\mathcal{G}_{F}$ in $\Delta_{q}$. Consequently, $\dot{\alpha} \geq \operatorname{cl}_{p} j(\mathscr{F})$. Since $j(\mathscr{F}) \rightarrow[\dot{x}]$ in $\left(X^{*}, p\right)$, the assumption that $\left(X^{*}, p\right)$ is $T_{3}$ is contradicted.

THEOREM 3.2. The following statements about a $T_{3}$ convergence space $(X, q)$ are equivalent.

(a) $(X, q)$ is r-bounded.

(b) $\left(X, \mathscr{D}_{q}\right)$ has $T_{3}$ completion.

(c) $[q]$ contains a coarsest $C_{3}$ member.

(d) $[q]$ contains a coarsest $\mathrm{SC}_{3}$ member. 
Proof. (b) $\Rightarrow$ (a). This is Lemma 3.1.

(b) $\Rightarrow$ (c) and (d). This follows from the fact that $\mathscr{W}_{q}$ is the coarsest $T_{3}$ member of $[q]$ (Proposition 2.1, [7]), and any completion of $\left(X, \mathscr{Q}_{q}\right)$ is necessarily strict.

(a) $\Rightarrow$ (b). Let $Y=X \cup\{a\}$ and let $i: X \rightarrow Y$ be the identity injection. Let $p$ be the finest convergence structure on $Y$ such that $(X, q)$ is a subspace of $(Y, p)$ and $i(\mathcal{G}) \rightarrow a$ for all $\mathcal{G} \in \Delta_{q}$. If $\mathcal{G} \in \Delta_{q}$, then $\mathrm{cl}_{q} i(\mathcal{G})=$ $i\left(\mathrm{cl}_{q} \mathcal{G}\right) \cap \dot{a} \rightarrow a$ in $(Y, p)$. Also, the assumption of $r$-boundedness guarantees that $\operatorname{cl}_{p} i(\mathscr{F})=i\left(\operatorname{cl}_{q} \mathscr{F}\right)$ for all $\mathscr{F} \in \bigcup^{q}$. Considering $p$ as a complete Cauchy structure on $Y$, it follows that $((Y, p), i)$ is a $T_{3}$ completion of $\left(X, \mathscr{W}_{q}\right)$.

(c) $\Rightarrow$ (b). If $\Delta_{q}=\varnothing$, then $\mathscr{D}_{q}=\bigodot^{q}$ is the coarsest $C_{3}$ member of [q]. If $\Delta_{q} \neq \varnothing$, let $\mathscr{F} \in \Delta_{q}$. Define $\mathcal{C}_{\mathscr{F}}=\mathcal{C}^{q} \cup\{\mathcal{G} \in F(X)$ : there is $n \in N$ such that $\left.\mathcal{G} \geq \operatorname{cl}_{q}^{n} \mathscr{F}\right\}$. Then $\mathcal{C}_{\mathscr{F}} \in[q]$ and $\bigodot_{\mathscr{F}}$ is easily seen to be a $T_{3}$ Cauchy structure. Next we construct a $T_{3}$ one-point completion of $\left(X, \bigodot_{\mathscr{F}}\right)$. Let $Y=X \cup\{a\}$, and let $p$ be the finest convergence structure on $Y$ such that $(X, q)$ is a subspace of $(Y, p)$ and $i(\mathcal{G}) \rightarrow a$ for all $\mathcal{G} \geq \operatorname{cl}_{q}^{n} \mathscr{F}$ for some $n \in N$. It is easy to verify that $((Y, p), i)$ is a $T_{3}$ completion of $\left(X, \mathcal{C}_{\mathscr{F}}\right)$. Since $\mathscr{D}_{q}$ is the infimum in $[q]$ of $\left\{\mathcal{C}_{\mathscr{F}}: \mathscr{F} \in \Delta_{q}\right\}$, it follows that $\mathscr{D}_{q}$ is the coarsest $C_{3}$ member of [q], and (b) is established.

(d) $\Rightarrow(b)$. The preceding proof is applicable here, since the $T_{3}$ completion constructed for each $\left(X, \bigodot_{\mathscr{F}}\right)$ is strict.

A Tychonoff topological space has a coarsest compatible uniformity iff the space is locally compact. Thus one would expect $r$-boundedness to be closely related to local compactness. This relationship is described in the next two propositions.

Proposition 3.3. A locally compact $T_{3}$ convergence space $(X, q)$ is $r$-bounded.

Proof. Let $\mathscr{F} \rightarrow x$ in $(X, q)$, and let $A$ be a compact set in $\mathscr{F}$. It follows easily that each $\mathcal{G} \in \Delta_{q}$ contains a set $G_{\mathcal{G}}$ which is disjoint from $A$. If $M=\cup\left\{G_{\mathcal{G}}: \mathcal{G} \in \Delta_{q}\right\}$, then $M \cap A=\varnothing$, and therefore $\mathscr{F} \vee \mathfrak{N}_{q}=\varnothing$, which establishes that $(X, q)$ is $r$-bounded. 
As in [5] and [7], we define a convergence space to be almost topological if it has the same ultrafilter convergence as a topological space.

Proposition 3.4. If $(X, q)$ is a $T_{3}$, almost topological space, then $(X, q)$ is locally compact iff $(X, q)$ is $r$-bounded.

Proof. If $(X, q)$ is not locally compact, then there is $\mathscr{F} \rightarrow x$ such that each $F \in \mathscr{F}$ is a member of a non-convergent ultrafilter $\mathcal{G}_{F}$. If $\mathrm{cl}_{q}\left(\mathcal{G}_{F}\right) \vee \mathscr{H C}$ $\neq \varnothing$ for some filter $\mathcal{H} \rightarrow y$, then $y$ is an adherent point of $\mathrm{cl}_{q}\left(\mathcal{G}_{F}\right)$; since $(X, q)$ is assumed to be almost topological, $\mathcal{G}_{F} \rightarrow y$, contrary to assumption. Thus $\mathcal{G}_{F} \in \Delta_{q}$ for all $F \in \mathscr{F}$, and so $\mathscr{F} \vee \mathscr{T}_{q} \neq \varnothing$. It follows that $(X, q)$ is not $r$-bounded.

We next define a $T_{3}$ convergence space $(X, q)$ to be $s$-bounded if, for all $\mathcal{G} \in \Delta_{q}, \mathcal{G} \vee\left(\cap \mathbb{Q}_{\mathcal{G}}\right)=\varnothing$, where $\mathbb{Q}_{\mathcal{G}}=\left\{\mathscr{F} \in \Delta_{q}\right.$ : for all $n \geq 1,\left(\operatorname{cl}_{q}^{n} \mathscr{F}\right)$ $\left.\vee\left(\operatorname{cl}_{q}^{n} \mathcal{G}\right)=\varnothing\right\}$.

Proposition 3.5. If a $T_{3}$ space $(X, q)$ contains a closed, infinite, discrete subset $A$, then $(X, q)$ is not s-bounded.

Proof. Any ultrafilter $\mathcal{G}$ on $X$ which contains $A$ is a closed member of $\Delta_{q} . \mathbb{Q}_{\mathcal{G}}$ contains all free ultrafilters on $X$ which contain $A$ and are distinct from $\mathcal{G}$. Thus $\mathcal{G} \vee\left(\cap \mathbb{Q}_{\mathcal{G}}\right) \neq \varnothing$, so $(X, q)$ is not $s$-bounded.

THEOREM 3.6. For a $T_{3}$ convergence space $(X, q)$, the following are equivalent.

(a) $(X, q)$ is both $r$-bounded and s-bounded.

(b) Each $T_{3}$ member of $[q]$ is $C_{3}$.

(c) Each $T_{3}$ member of $[q]$ is $S C_{3}$.

Proof. (b) $\Rightarrow$ (a). $(X, q)$ is $r$-bounded by Lemma 3.1. Let $\mathcal{G} \in \Delta_{q}$ and define $\Re_{\mathcal{G}}$ to be the set of all filters on $X$ which are finer than some finite intersection of members of $\mathbb{Q}_{\mathcal{G}}$. Let $\mathscr{D}_{\mathcal{G}}=e^{q} \cup \mathcal{C}_{\mathcal{G}} \cup \mathscr{Q}_{\mathcal{G}}$, where $\bigodot_{\mathcal{G}}=\{\mathscr{F} \in$ $F(X)$ : there is $n \in N$ such that $\left.\mathscr{F} \geq \mathrm{cl}_{q}^{n} \mathcal{G}\right\}$. It can be shown by direct arguments that $\mathscr{D}_{\mathcal{G}}$ is a $T_{3}$ member of $[q]$. If $\mathscr{F} \in \mathbb{Q}_{\mathcal{G}}$, then [Fु $]=\mathbb{Q}_{\mathcal{G}}$. Since $[\mathcal{G}] \neq[\mathscr{F}]$, it must be true that $\mathcal{G} \vee\left(\cap \mathbb{Q}_{\mathcal{G}}\right)=\varnothing$; otherwise $[\dot{\mathscr{F}}] \geq \Sigma \mathcal{G}$, so $[\dot{\mathscr{F}}] \rightarrow[\mathcal{G}]$ in $\left(X^{*}, \mathcal{C}_{r}^{*}\right)$, contrary to Proposition 1.2. Thus $(X, q)$ is $s$-bounded.

(c) $\Rightarrow$ (b). Obvious. 
(a) $\Rightarrow$ (c). Assume $(X, q)$ is both $r$-bounded and $s$-bounded, and let $\mathcal{e} \in[q]$ be $T_{3}$. By Proposition 2.1 it is sufficient to show that $\left(X^{*}, q_{1}^{*}\right)$ is $T_{3}$; recall that $\mathcal{H} \rightarrow[\mathcal{F}]$ in $q_{1}^{*}$ iff there is $\mathcal{G} \in[\mathcal{F}]$ such that $\mathcal{H} \geq \Sigma \mathcal{G}$.

If $\Sigma \mathscr{F} \rightarrow[\dot{x}]$ in $\left(X^{*}, q_{1}^{*}\right)$, then $\mathscr{F} \rightarrow x$ in $(X, q)$ and also $\mathrm{cl}_{q} \mathscr{F} \rightarrow x$. Using $r$-boundedness, one can show that $\operatorname{cl}_{q_{1}^{*}}(\Sigma \mathscr{F})=\Sigma\left(\mathrm{cl}_{q} \mathscr{F}\right)$, and the latter $q_{1}^{*}$-converges to $[\dot{x}]$. If $\mathcal{G} \in \mathcal{C}-\mathcal{C}^{q}$, then $\mathcal{G} \in \Delta_{q}$ and the two types of boundedness can be used to show that $\mathrm{cl}_{q_{1}^{*}} \Sigma \mathcal{G}=\Sigma\left(\mathrm{cl}_{X} \mathcal{G}\right) \cap[\dot{\mathcal{G}}$ ]; again, the latter filter $q_{1}^{*}$-converges to [G]. Thus $\left(X^{*}, q_{1}^{*}\right)$ is regular. The $T_{3}$ property is established by showing, in addition, that $\mathrm{cl}_{q_{1}^{*}}\{[\mathcal{H C}]\}=\{[\mathcal{H C}]\}$ for all $\mathcal{H} \in \mathcal{C}$; this again follows direct from $r$-boundedness and $s$-boundedness.

To simplfy the terminology, we shall say that a $T_{3}$ convergence space satisfying any of the three equivalent conditions of Theorem 3.6 is $r s$-bounded. The next corollary is an immediate consequence of the preceding theorem, Propositions 3.4 and 3.5, and the fact that a $T_{3}$ topological space which is not countably compact contains an infinite, closed, discrete subset.

Corollary 3.7. An rs-bounded $T_{3}$ topological space is both locally compact and countably compact.

COROllaRY 3.8. A metrizable space is rs-bounded iff it is compact.

Proof. If $(X, q)$ is compact, then $[q]=\left\{\complement^{q}\right\}$, and $\bigodot^{q}$ is obviously $C_{3}$. The converse follows from Corollary 3.7.

\section{REFERENCES}

[1] R. N. Ball, Convergence and Cauchy structures on lattice ordered groups, Trans. Amer. Math. Soc., 259 (1980), 357-392.

[2] R. Fric and D. C. Kent, Completion functors for Cauchy spaces, Internat. J. Math. \& Math. Sci., 4 (1979), 589-604.

[3] H. H. Keller, Die Limes-Uniformisierbarkeit der Limesräume, Math. Ann., 176 (1968), 334-341.

[4] D. C. Kent and G. D. Richardson, The decomposition series of a convergence space, Czech. Math. J., 23 (1973), 437-446.

[5] __ Regular completions of Cauchy spaces, Pacific J. Math., 51 (1974), 483-490.

[6] Completely regular and w-regular spaces, Proc. Amer. Math. Soc., 82 (1981), 649-652.

[7] D. C. Kent, A note on regular Cauchy spaces, Pacific J. Math., 94 (1981), 333-339.

[8] K. McKennon, The strict topology and the Cauchy structure of the spectrum of a $C^{*}$-algebra, General Topology Appl., 5 (1975), 249-262. 
[9] J. Ramaley and O. Wyler, Cauchy spaces II. Regular completions and compactifications, Math. Ann., 187 (1970), 187-199.

[10] E. E. Reed, Completions of uniform convergence spaces, Math. Ann., 194 (1971), 83-108.

Received March 15, 1982.

WASHington State UNIVERSITY

Pullman, WA 99164

AND

EAST CAROLINA UNIVERSITY

GREENVILLE, NC 27834 


\section{PACIFIC JOURNAL OF MATHEMATICS}

EDITORS

Donald BabBITT (Managing Editor)

University of California

Los Angeles, CA 90024

Hugo Rossi

University of Utah

Salt Lake City, UT 84112

C. C. Moore and Arthur Ogus

University of California

Berkeley, CA 94720
J. DugunduI

Department of Mathematics

University of Southern California

Los Angeles, CA 90089-1113

R. FINN and H. SAMELSON

Stanford University

Stanford, CA 94305

\section{ASSOCIATE EDITORS}
R. ARENS
E. F. BECKENBACH
B. H. NeumanN
F. WolF
K. YosHIDA (1906-1982)

\section{SUPPORTING INSTITUTIONS}

UNIVERSITY OF ARIZONA

UNIVERSITY OF BRITISH COLUMBIA

CALIFORNIA INSTITUTE OF TECHNOLOGY

UNIVERSITY OF CALIFORNIA

MONTANA STATE UNIVERSITY

UNIVERSITY OF NEVADA. RENO

NEW MEXICO STATE UNIVERSITY

OREGON STATE UNIVERSITY
UNIVERSITY OF OREGON

UNIVERSITY OF SOUTHERN CALIFORNIA

STANFORD UNIVERSITY

UNIVERSITY OF HAWAII

UNIVERSITY OF TOKYO

UNIVERSITY OF UTAH

WASHINGTON STATE UNIVERSITY

UNIVERSITY OF WASHINGTON 


\section{Pacific Journal of Mathematics}

\section{Vol. 111, No. $1 \quad$ November, 1984}

Harald Brandenburg and Adam Stefan Mysior, For every Hausdorff

space $Y$ there exists a nontrivial Moore space on which all continuous

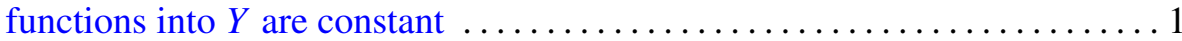

Henry Dappa, A Marcinkiewicz criterion for $L^{p}$-multipliers $\ldots \ldots \ldots \ldots \ldots 9$

P. H. Doyle, III and John Gilbert Hocking, Bijectively related spaces. I.

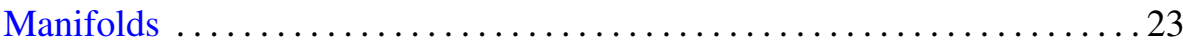

Joel Hass, Complete area minimizing minimal surfaces which are not totally

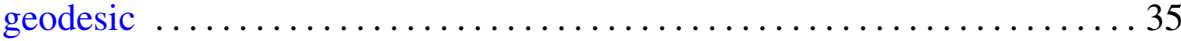

Aarno Hohti, On Ginsburg-Isbell derivatives and ranks of metric spaces .... 39

Richard Howard Hudson, Diophantine determinations of $3^{(p-1) / 8}$ and $5^{(p-1) / 4}$

A. F. Izé and A. Ventura, Asymptotic behavior of a perturbed neutral functional-differential equation related to the solution of the unperturbed

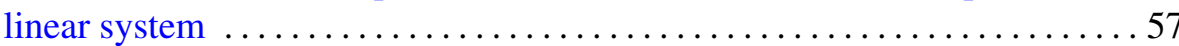

Palle E. T. Jorgensen, Spectral representations of unbounded nonlinear

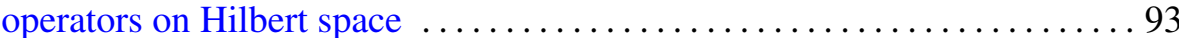

Darrell Conley Kent and Gary Douglas Richardson, Cauchy spaces with

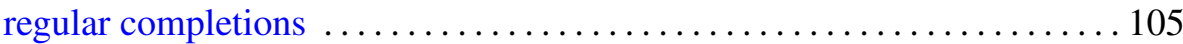

Mark Mahowald, An addendum to: "bo-resolutions" ................ 117

Stuart Wayne Margolis and Jean-Eric Pin, Minimal noncommutative varieties and power varieties

Carla Massaza and Alfio Ragusa, Some conditions on the homology groups of the Koszul complex

Vicente Miquel Molina, Some examples of Riemannian almost-product manifolds

Roderic Murufas, Inverse spectral problems for certain differential operators

Ulrich Oertel, Closed incompressible surfaces in complements of star links

Katsuro Sakai, A characterization of local equiconnectedness

William Victor Smith and Don Harrell Tucker, Weak integral convergence theorems and operator measures 